\title{
The small GTPase $R$ heb is required for spermatogenesis but not oogenesis
}

\author{
M D Baker ${ }^{1,+}$, M Ezzati ${ }^{2}$, G M Aloisio ${ }^{1}$, E D Tarnawa ${ }^{2}$, I Cuevas ${ }^{1}$, Y Nakada ${ }^{1}$ and D H Castrillon ${ }^{1}$ \\ ${ }^{1}$ Department of Pathology and ${ }^{2}$ Division of Reproductive Endocrinology and Infertility, Department of Obstetrics \\ and Gynecology, University of Texas Southwestern Medical Center, 6000 Harry Hines Boulevard, Dallas, \\ Texas 75390-9072, USA
}

Correspondence should be addressed to D H Castrillon; Email: Diego.Castrillon@UTSouthwestern.edu

${ }^{+} \mathrm{M}$ D Baker is now at Fort Worth Fertility, 1800 Mistletoe Blvd, Fort Worth, Texas 76104, USA

\begin{abstract}
The process of germ cell development is under the tight control of various signaling pathways, among which the PI3K-Akt-mTOR pathway is of critical importance. Previous studies have demonstrated sex-specific roles for several components of this pathway. In the current study, we aimed to evaluate the role of Rheb, a member of the small GTPase superfamily and a critical component for mTORC1 activation, in male and female gametogenesis. The function of Rheb in development and the nervous system has been extensively studied, but little is known about its role in the germ line. We have exploited genetic approaches in the mouse to study the role of Rheb in the germ line and have identified an essential role in spermatogenesis. Conditional knockout (cKO) of $R$ he $b$ in the male germ line resulted in severe oligoasthenoteratozoospermia and male sterility. More detailed phenotypic analyses uncovered an age-dependent meiotic progression defect combined with subsequent abnormalities in spermiogenesis as evidenced by abnormal sperm morphology. In the female, however, germ-cell specific inactivation of Rheb was not associated with any discernible abnormality; these cKO mice were fertile with morphologically unremarkable ovaries, normal primordial follicle formation, and subsequent follicle maturation. The absence of an abnormal ovarian phenotype is striking given previous studies demonstrating a critical role for the mTORC1 pathway in the maintenance of primordial follicle pool. In conclusion, our findings demonstrate an essential role of $R$ heb in diverse aspects of spermatogenesis but suggest the existence of functionally redundant factors that can compensate for Rheb deficiency within oocytes. Reproduction (2014) 147 615-625
\end{abstract}

\section{Introduction}

The $\mathrm{PI} 3 \mathrm{~K} / \mathrm{AKT} / \mathrm{mTOR}$ signaling pathway is highly conserved among diverse species and plays important roles in the regulation of cell growth. Among other functions, this pathway serves as a key mediator for cellular responses to both the environment and the intracellular milieu, including growth factors, nutrient availability, oxygen levels, and intracellular energy charge (Laplante \& Sabatini 2009). Working through this pathway, these environmental and intracellular signals exert many changes in cellular physiology to maintain homeostasis. Among these, changes in the rate of protein translation and turnover, and cell-cycle progression, are among the best characterized (Laplante \& Sabatini 2009).

The cell survival and growth kinase PI3K represents one important node of this pathway. PI3K converts membrane-bound phosphatidylinositol 4,5-bisphosphate (PIP2) to phosphatidylinositol (3,4,5)-trisphosphate (PIP3). PIP3 acts as a second messenger by membranerecruitment and activation of the serine-threonine kinase Akt. Pten, a powerful inhibitor of PI3K signaling, acts to convert PIP3 back to PIP2, thereby suppressing Akt activation. Akt in turn phosphorylates and thereby inhibits Tsc2, and also phosphorylates many additional downstream targets including the Foxo transcription factors, which also play multiple roles in cell growth and proliferation (John et al. 2008, Goertz et al. 2011, Tzivion et al. 2011). The AMP kinase (AMPK) is another important node of this pathway, acting via Tsc2. AMPK is activated by energy stress/low nutrient levels (which result in high intracellular levels of AMP) and also phosphorylates Tsc2. Tsc2 can also be phosphorylated by additional kinases (e.g. Redd1, Erk, and Ikk $\beta$ ) in response to other environmental cues (Laplante \& Sabatini 2009). Tsc2 and its physical partner, Tsc1, are structurally unrelated but form an obligate complex whose principal (and indeed, only well-documented) role is as negative regulator (via Rheb) of mTORC1 (one of the two physically and functionally distinct signaling complexes in which mTOR is a key component, the other being mTORC2). The only known function of Tsc1 is to stabilize Tsc2 and prevent its degradation. 
Rheb is a small GTPase and member of the Ras family and is ubiquitously expressed in mammals. Tsc2 contains a GAP (GTPase-activating) domain that stimulates the intrinsic GTPase activity of Rheb, thereby promoting the conversion of Rheb-GTP into Rheb-GDP. Whereas Rheb-GTP potently activates mTORC1, RhebGDP is inactive. Rheb directly activates mTORC1 through mechanisms that are not well understood. These findings are based on numerous genetic and biochemical studies performed in vertebrate and vertebrate model systems and appear to be universal. For example, in TsC1 or Tsc2 mutant cells, mTORC1 is constitutively active, potently stimulating cell growth (Fingar \& Blenis 2004, Zou et al. 2011).

mTORC1 in turn regulates protein translation largely through two proteins that are key components of the translational control machinery, ribosomal protein S6 (S6) and 4E-binding protein (4EBP), to potently drive protein translation and cell growth. Phosphorylation of p70S6 kinase (S6K) results in the activation of S6, which allows for the formation of the mRNA translation machinery. mTORC1 also increases protein synthesis by inactivating the translational repressor 4EBP which binds to and inhibits the eukaryotic translation initiation factor 4E (Laplante \& Sabatini 2009).

Several studies have implicated some of these key components of the PI3K/AKT/mTOR pathway in spermatogenesis and oogenesis. Foxo1 is specifically expressed in undifferentiated spermatogonia, and the three Foxos together regulate multiple steps of spermatogenesis including stem cell maintenance and differentiation (Goertz et al. 2011, Tarnawa et al. 2013). Pten is also required for spermatogenesis; its inactivation with the germ line-specific Vasa-Cre ( $\operatorname{Tg}(\mathrm{Dd} \times 4$-cre $) 1 D$ cas $)$; Gallardo et al. 2007a) results in severe defects in spermatogonial maintenance and differentiation, at least in part through Foxo1 (Goertz et al. 2011). Other studies have implicated mTORC1 as a regulator of spermatogenesis and spermatogonial stem cell maintenance (Hobbs et al. 2010).

In the ovary, Foxo3 is specifically expressed in primordial oocytes, where it serves to potently restrain their activation. In Foxo3-null mice, primordial follicles develop normally, but undergo global activation immediately after they are formed, resulting in a syndrome of deregulated follicle growth and premature female infertility (Castrillon et al. 2003, Hosaka et al. 2004, John et al. 2007 , 2008). Strikingly, an identical ovarian phenotype occurs following conditional germ line inactivation of three other PI3K/AKT/mTOR pathway components: Pten, $T_{S C 1}$, and TSC2, underscoring the importance of this pathway in the maintenance of the female germ line (John et al. 2008, Reddy et al. 2008, Adhikari et al. 2009, 2010). Pten inactivation results in global primordial follicle activation through Akt and consequent phosphorylation and cytoplasmic sequestration/inactivation of Foxo3 (John et al. 2008). TsC1 and TsC2 inactivation in oocytes results in global primordial activation associated with mTOR pathway hyperactivity and increased phosphorylation of S6K and 4EBP within the oocytes (Adhikari et al. 2009, 2010). These recent mouse models have laid the groundwork for uncovering the regulation of primordial follicle maintenance, but further investigations are required to identify how these canonical PI3K branch components (Pten, Akt, and Foxos) interact with canonical mTOR branch components (Tsc1/Tsc2) (Sullivan \& Castrillon 2011). To further explore these questions and the role of mTOR signaling in gametogenesis, we studied the impact of Rheb inactivation in both the male and female germ line.

\section{Materials and methods}

\section{Mouse strains, breeding, and tissue processing and expression analyses}

This study was approved by the University of Texas Southwestern Medical Center Institutional Animal Care and Use Committee. Generation of both the Rheb-floxed and Vasa-Cre alleles and genotyping protocols were previously described (Gallardo et al. 2007a, Zou et al. 2011). Vasa-Cremales were crossed with Rhe $b^{f / f}$ females. Vasa-Cre; $R$ he $b^{f /+}$ male offspring were again crossed to $R h e b^{f / f}$ females to produce Vasa-Cre; $R h e b^{f /-}$ experimental mice. Matched Vasa-Cre negative siblings were used as controls. All tissues were processed immediately after procurement. The tissues were fixed in $10 \%$ neutral buffered formalin for $24 \mathrm{~h}$, embedded in paraffin, and cut into $5-\mu \mathrm{m}$ sections for immunohistochemistry (IHC) or hematoxylin and eosin (H\&E) staining. Tissue sections from experimental and control samples were placed on the same slide to ensure identical processing. Expression analyses by digital Northern were conducted as previously described (Gallardo et al. 2007 b).

\section{Sperm analysis and breeding assays}

A complete sperm analysis was performed to quantify the output of the testis. Epididymal sperm density was obtained by counting the number of hematoxylin-stained sperm heads in three $20 \times$ fields for each epididymis. The area in the seminiferous tubule was calculated using the Image) (Bethesda, MD, USA) Software package (Schneider et al. 2012). Data were graphed as total sperm per square pixel. Epididymal sperm concentration was used as a secondary measure of total sperm output. One epididymis was dissected and placed into $1 \mathrm{ml}$ of PBS. Approximately five cuts were made in the epididymis followed by $30 \mathrm{~min}$ incubation at $37^{\circ} \mathrm{C}$ to release the sperm from the tissue. Sperm concentration was then measured using a fixed cover slip counting chamber (Cell-Vu, DRM-600). Motility was also noted, but experimental samples contained too few sperm for percentages to be accurately calculated. To study sperm morphology, a small drop of the sperm suspension was placed on a glass microscope slide and spread evenly across the slide with a cover slip. The sperm smear was allowed to dry and was then subjected to Diff-Quik staining (Fisher Scientific, Pittsburgh, PA, USA; 65044C). Previously published criteria were used for the determination of normal sperm 
morphology (Wyrobek \& Bruce 1975). Breeding assays were performed as previously described (Gallardo et al. 2008). Three-week-old experimental and sibling control males were placed with two 6-week-old FVB females and observed for 20 weeks. Females were placed with one FVB male and observed for 18 weeks.

\section{Immunohistochemistry and quantitative analyses of morphology in tissue sections}

Slides were deparaffinized in xylene, hydrated in an ethanol series, subjected to antigen retrieval by boiling in $10 \mathrm{mM}$ sodium citrate $(\mathrm{pH}$ 6.0) for $15 \mathrm{~min}$, and cooled at room temperature (RT) for $20 \mathrm{~min}$. After peroxidase blocking (3\% hydrogen peroxide in water) for $30 \mathrm{~min}$, slides were washed in water and then blocked in BSA (1\% in $1 \times$ PBS) for $15 \mathrm{~min}$. The slides were then incubated with primary antibody overnight at $4{ }^{\circ} \mathrm{C}$, subjected to a TBST $(1 \mathrm{M}$ Tris- $\mathrm{HCl}, 5 \mathrm{M} \mathrm{NaCl}$, and $1 \times$ Tween-20) wash series, incubated with secondary antibody for $30 \mathrm{~min}$ at RT (Immpress; Vector Laboratories (Burlington, CA, USA)), and subjected to a second TBST wash series. Signal was detected using a DAB liquid chromogen substrate kit (DAKO, Carpinteria, CA, USA). Slides were then counterstained with hematoxylin, rinsed in water, and air-dried.
Antibodies and titers used included Foxo1 (rabbit monoclonal; Cell Signaling Technology (Danvers, MA, USA), \#2880; 1:500), Crem1 (rabbit polyclonal; Santa Cruz Biotechnology, \#SC-440; 1:1000), mTOR (rabbit monoclonal; Cell Signaling Technology, \#2983; 1:1000), p-mTOR (rabbit monoclonal; Cell Signaling Technology, \#5536; 1:1000), p-4EBP (rabbit monoclonal; Cell Signaling Technology, \#9455; 1:1000), p-S6K (rabbit monoclonal; Cell Signaling Technology, \#9234; 1:1000), p-S6 (rabbit monoclonal; Cell Signaling Technology, \#4857; 1:1000), $\mathrm{pH} 2 \mathrm{AX}$ (rabbit monoclonal; Cell Signaling Technology, \#9718; 1:200 000), Ki67 (rabbit monoclonal; Thermo Scientific (Waltham, MA, USA), \#RM-9106-FO; 1:500), germ cell nuclear antigen (1:200 IHC) (courtesy of George Enders, University of Kansas). Cell counts were performed to quantify the four principal cellular stages of sperm development (spermatogonia, spermatocytes, round spermatids, and elongated spermatids). Counts for undifferentiated spermatogonia and round spermatids were facilitated by immunostaining these cell types with Foxo1 and Crem1 respectively, as described in the text. Spermatocytes and elongated spermatids were identified and counted based on their distinct morphology in routinely stained histological sections. For each cell type, a minimum of 20 tubules (staged from IV to VII) were counted per mouse.
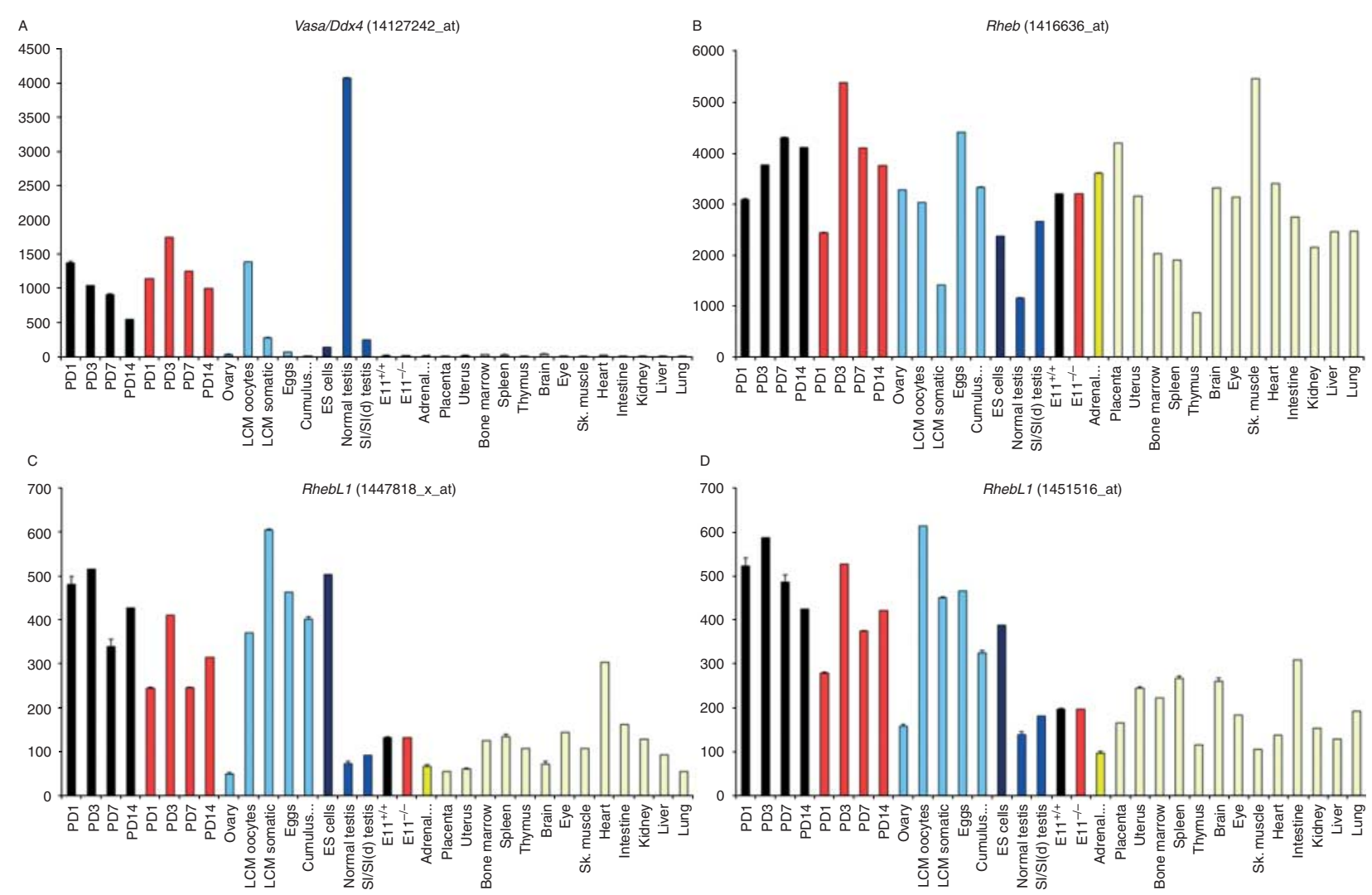

Figure 1 RNA expression analysis of Rheb and RhebL1. Graphs show relative expression levels of single probe set for indicated gene across multiple samples; error bars represent S.E.M. Samples are (left to right) Foxo $3^{+/+}$PD1, 3, 7, and 14 ovaries (black), Foxo3 ${ }^{-1-}$ PD1, 3, 7, 14 ovaries (red), adult ovary, laser-capture microdissected (LCM) primary and secondary oocytes, LCM somatic cells (primary plus secondary granulosa cells and surrounding stroma), eggs, cumulus cells, ES cells, normal adult testis, SI/SId (germ cell-depleted) adult testis, Foxo3 ${ }^{+/+}$E11 embryos, Foxo3 ${ }^{-/-}$E11 embryos, adrenal gland, placenta, uterus, bone marrow, spleen, thymus, brain, eye, skeletal muscle, heart, intestine, kidney, liver, and lung. (A) $D d x 4$ (Vasa) as representative control (germ cell-specific gene in both males and females). (B) Rheb. (C) Rheb/1 (probe set \#1). (D) Rheb/1 (probe set \#2). 


\section{Statistical analysis}

Data were graphed and analyzed using GraphPad Prism 5 (La Jolla, CA, USA). Two-tailed Student's t-tests were used to evaluate significance and calculate $P$ values, with threshold values as described in the results or figure legends. Error bars represent S.E.M. values. A $P$ value of $<0.05$ was considered significant.

\section{Results}

\section{Expression of Rheb and Rhebl1 in the gonad and the adult germ line}

We first characterized the expression of Rheb and its closest homolog in the murine genome, Rheb/1 ( $52 \%$ identical and $73 \%$ similar at the amino acid level). We took advantage of a previously described microarrybased platform for digital Northern analysis of reproductive tissues and cell types (Gallardo et al. $2007 b$, Contreras et al. 2010). The samples subjected to expression profiling included WT and Foxo3-null ovaries at postnatal day (PD) 1, 4, 7, and 14 (selected because primordial follicles make up a disproportionately greater fraction of the ovarian mass early in life, leading to good representation of primordial follicle transcripts). In addition, laser-capture microdissected (LCM) oocytes and surrounding granulosa cells from primary to secondary follicles (i.e. early growing follicles) were included given the important role of the mTOR/PI3K pathway in early follicle growth (John et al. 2008, Adhikari et al. 2009, 2010). Other samples included superovulated eggs, ES cells, normal testis, germ cell-deficient $\mathrm{SI} / \mathrm{SI}(\mathrm{d})$ testes, embryonic day 11 (E11) embryos, and diverse adult tissues (Fig. 1).

$D d x 4$ (a.k.a Vasa) was included as a representative germ cell-specific control validating the samples and methodology. Ddx4 was expressed in all PD1-14 ovarian samples and in LCM oocytes, but was expressed at low (nearly baseline) levels in the adult ovary, consistent with the fact that oocytes make up only a very small percentage of the ovarian mass (Gallardo et al. 2007b). Among adult tissues, $D d x 4$, was expressed at high levels only in the testis (only tall peak in the right half of the panel) but nearly absent in germ cell-deficient testes, as expected given $D d x 4^{\prime}$ s germ cell-specific expression. In contrast, Rheb was much more broadly expressed with high expression across all tissues, including ovaries and testis, where it appears to be expressed in both the germ line and somatic cells. In contrast, Rheb/1 was expressed at lower apparent levels overall (with lower signal strengths), but like Rheb was also very broadly expressed. Although Rheb was represented on the array by a single probe, Rheb/1 was represented by two separate probes. Note that the expression peaks from the second probe are virtually superimposable on the first, consistent with faithful readout of gene expression levels across probes. Neither Rheb nor Rheb/1 were differentially expressed in Foxo3- null ovaries (red bars), arguing that neither genes is a direct Foxo3 target. Thus, in summary, Rheb is expressed in gonads and the female germ line, making it a plausible candidate for a biological role in the germ line.

\section{Conditional inactivation of Rheb in the germ line with Vasa-Cre}

A floxed Rheb allele $\left(R h e b^{f}\right)$ was crossed with the germ cell-specific Cre recombinase, Vasa-Cre. This strategy allows for prenatal deletion of Rheb in both the male and female germ lines, as Vasa-Cre is expressed in both male and female germ cells beginning at E15 (Gallardo et al. 2007a). Available PCR genotyping protocols permit specific amplification of the WT, floxed, and null (i.e. Cre-recombined) Rheb alleles. Confirmation of Vasa-Cre-mediated excision of the floxed third exon of the Rheb locus was performed through PCR genotyping of both the Rheb-floxed and Rheb-null alleles in Vasa-Cre, Rhe ${ }^{f /+}$ mice. Both the WT and floxed Rheb alleles were detected in multiple tissues of Vasa-Cre; $R h e b^{f /+}$ mice: however, the floxed allele was clearly less abundant in the testis, consistent with germ cell Cre recombination and Rheb inactivation (Fig. 2A). The Rheb

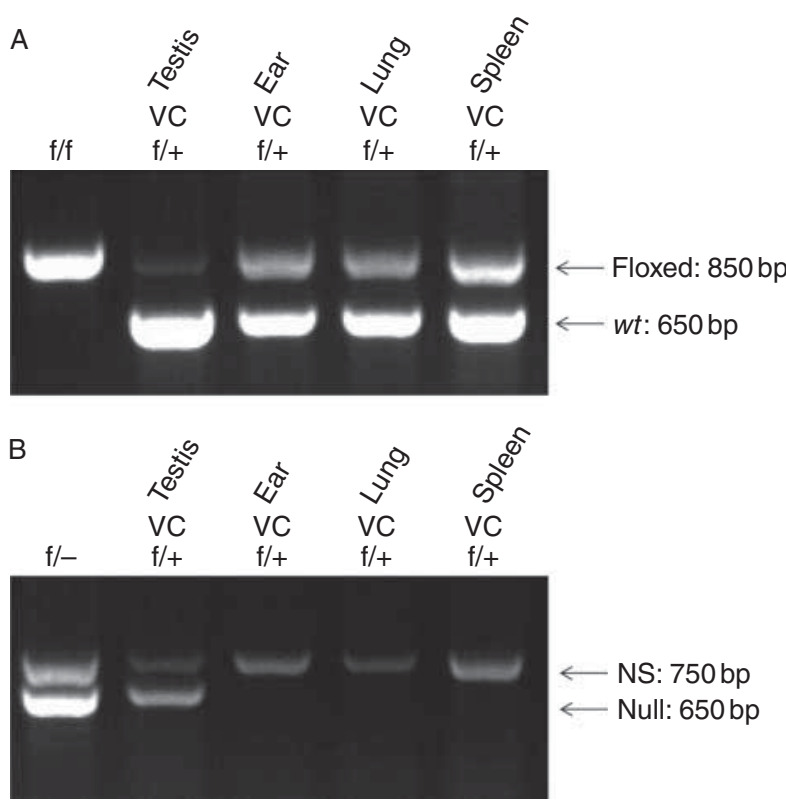

Figure 2 Genotype confirmation of germ cell Rheb conditional knockout. (A) An ethidium bromide-stained 1\% agarose gel shows the presence of the Rheb-floxed allele $(850 \mathrm{bp})$ in tail DNA from a $R h e b$ homozygous-floxed mouse as well as all other tissues of a Vasa-Cre; $R h e b^{f /+}$ (heterozygous) male. The floxed (f) band is decreased in the Vasa-Cre; $R$ heb $b^{f /+}$ testis consistent with Vasa-Cre-mediated recombination in germ cells. The $w t(+)$ band (650 bp) is observed in tissues from this heterozygous male mouse, as expected. (B) Cre-mediated excision was further confirmed by the presence of the Rheb-null allele (650 bp) in a Vasa-Cre; $R h e b^{f /+}$ testis as well as a $R h e b^{f /-}$ control testis. The Rheb-null allele was not detected in other tissues (e.g. skin, lung, and spleen). NS, non-specific PCR product ( $\sim 50 \mathrm{bp})$. 
A

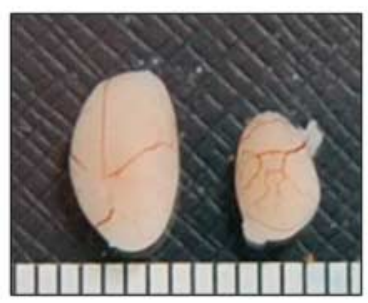

2 months

C
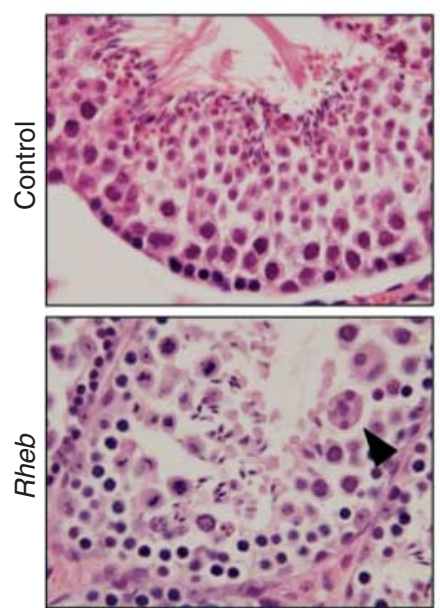

2 months

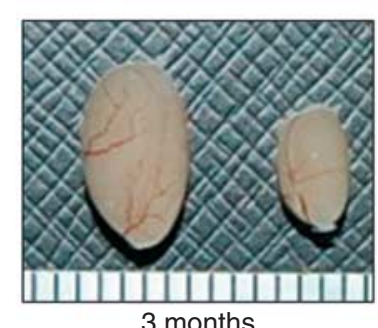

3 months
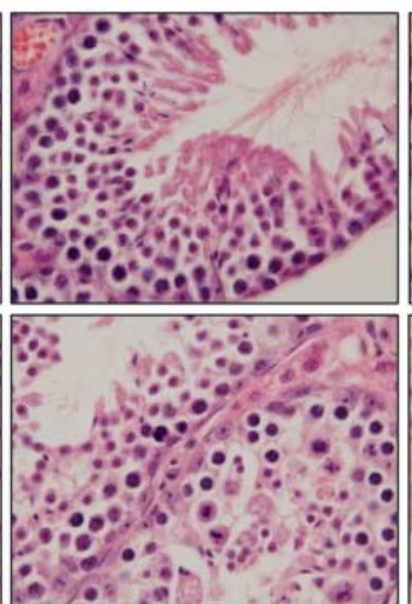

3 months
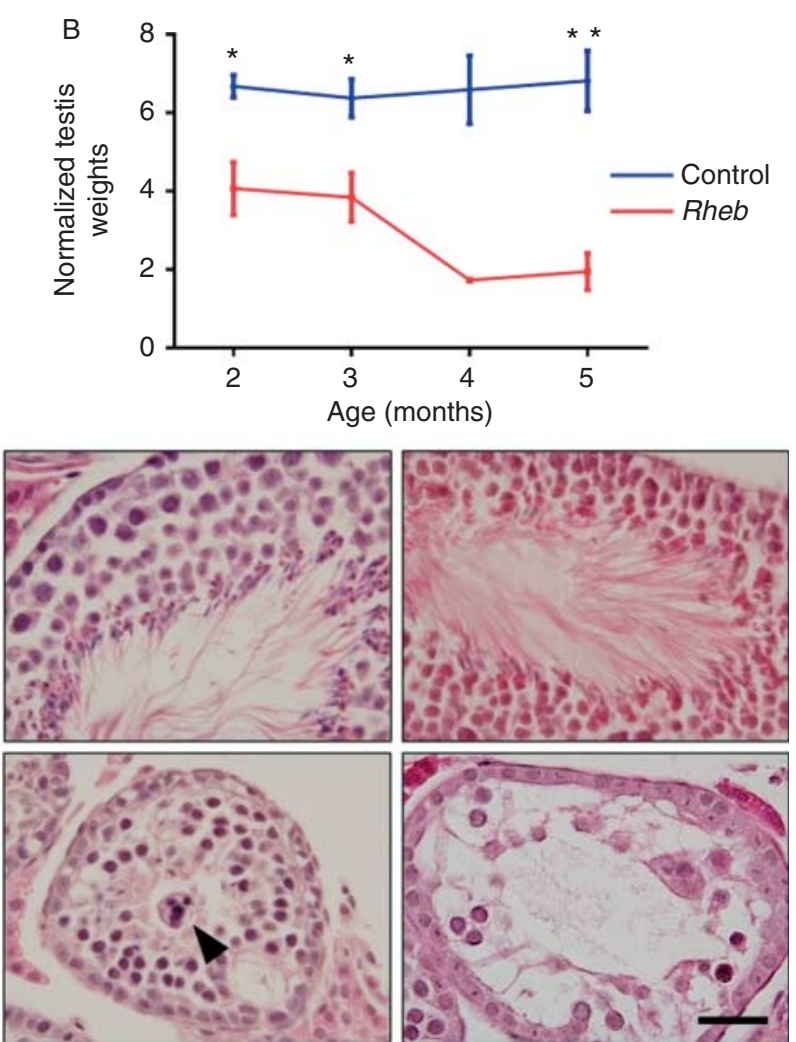

4 months

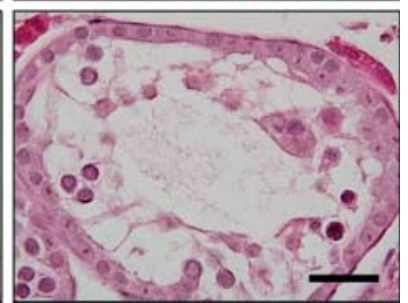

5 months

Figure 3 Conditional Rheb inactivation results in a progressive defect of spermatogenesis. (A) Intact testes. (Right) Vasa-Cre; Rhe $b^{f /-}$ and (left) $R h e b^{f /-}$ sibling control. Ruler markings, $1 \mathrm{~mm}$. (B) Testis weights in mice of different ages. Red, Vasa-Cre; Rhe $b^{f /-}$ and blue, Rhe $b^{f /-}$ sibling control. ${ }^{*} P<0.05$ and ${ }^{* *} P<0.005$, by unpaired $t$-test ( 2 months, $n=2 ; 3$ months, $n=3 ; 4$ months, $n=1$ (no statistical test performed); and 5 months, $n=3$ ) (C) H\&E stained tissue sections from testes of 2- to 5-month-old Rheb cKO and sibling control mice. Representative fields illustrate an abnormal organization of seminiferous tubules at the 2 -month time point and the age-dependent defect in meiotic progression resulting in the progressive loss of round and elongating spermatids in the 5-month-old testis. Arrowheads indicate large multinucleated germ cells. Bar $=50 \mu \mathrm{m}$.

null allele was detected in the testis of the Vasa-Cre; $R h e b^{f /+}$ mouse but not in other tissues (Fig. 2B) again consistent with germ cell-specific Cre activity. Germ line transmission of the Rheb-null allele was also confirmed by genotyping progeny from Vasa-Cre; $R$ he $b^{f /-}$ females crossed with WT males; all progeny carried the null allele $(n=18)$, confirming efficient, biallelic ablation of Rheb within oocytes.

\section{Gross and histological analyses of gonads from male and female Rheb conditional knockout mice}

By gross examination, adult Rheb conditional knockout (cKO; Vasa-Cre; $R$ heb ${ }^{f /-}$ ) testes were much smaller than those of Vasa-Cre-negative $\left(R h e b^{f /-}\right)$ sibling controls (Fig. 3A). A progressive atrophy of the testes was observed in experimental males. Vasa-Cre; $R h e b^{f /-}$ testis weights underwent a further twofold decrease between 3 and 5 months of age (Fig. 3B). Testis sections from 3-month-old Vasa-Cre; Rheb ${ }^{f /-}$ males exhibited hypospermatogenesis with all stages of sperm present. However, in aged mice the seminiferous tubules were more severely affected by the loss of Rheb. At 5 months of age, there was severely decreased spermatogenesis and while spermatocytes were present, very few round or elongated spermatids were observed (Fig. 3C). In contrast, Vasa-Cre; $R h e b^{f /-}$ ovaries were grossly unremarkable (Fig. 4A). Histological analysis of ovary sections showed the presence of growing follicles of all stages (Fig. 4B) and a healthy pool of quiescent primordial follicles (Fig. 4C).

\section{Rheb cKO males have decreased sperm counts and abnormal sperm morphology}

A complete sperm workup was performed to identify defects in total sperm count, morphology, and motility. Epididymides from both Vasa-Cre; Rhe $b^{t /-}$ and $R h e b^{f /-}$ controls were examined histologically for the presence of mature spermatozoa (Fig. 5A). Quantification of sperm head density clearly showed a progressive agedependent decrease in sperm production (Fig. 5B). This decrease was also quantified through epididymal sperm concentration counts (Fig. 5C). By 4 months of age, the 


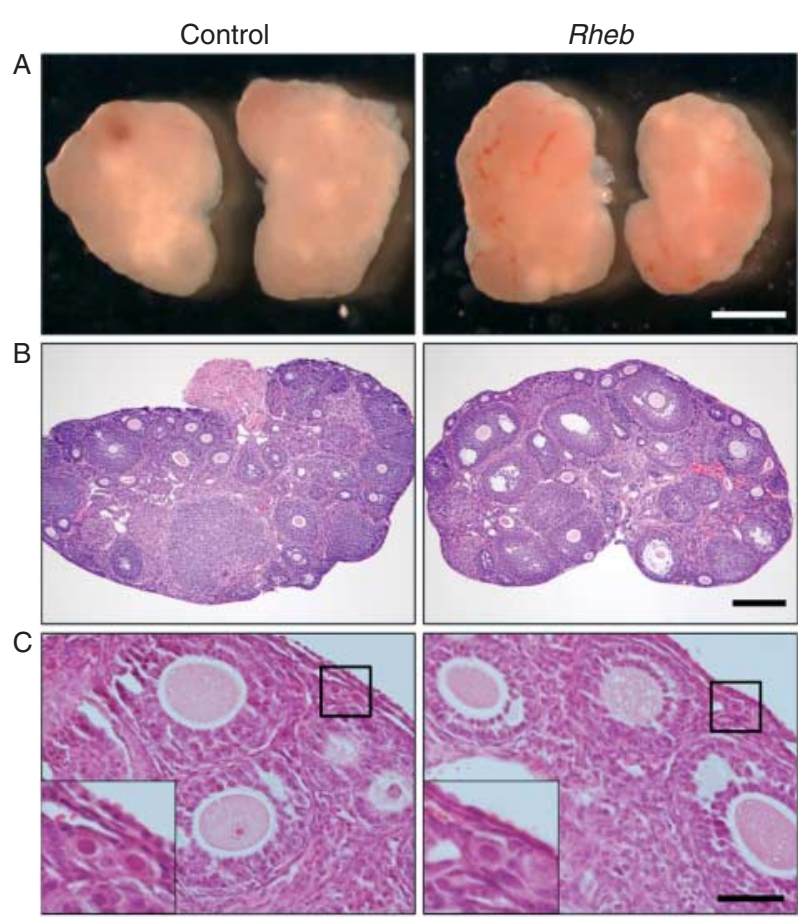

Figure 4 Ovaries of Rheb cKO mice are grossly normal and contain growing follicles of all stages, as well as morphologically normal primordial follicles. (A) Intact ovaries of Rheb cKO (Vasa-Cre; Rhe $b^{f /-}$ ) females were of similar size and shape relative to sibling $\left(R h e b^{f /-}\right)$ controls. Bar $=1 \mathrm{~mm}$. (B) H\&E stained tissue sections from Rheb cKO and sibling control ovaries revealed growing follicles of all stages. Bar $=250 \mu \mathrm{m}$. (C) Higher magnification shows growing primary and secondary follicles. Insets: morphologically normal, quiescent primordial follicles. Scale bar $=50 \mu \mathrm{m}$.
Vasa-Cre; $R$ heb ${ }^{f /-}$ males exhibited severe oligoasthenoteratozoospermia. The predominant morphological defect observed was amorphous head shapes (Fig. 5D).

\section{Rheb is required for male, but not female, fertility}

To quantify the effect of Rheb cKO on fertility, breeding assays were set up for Vasa-Cre; Rhe $b^{t /-}$ males and females and $R h e b^{t /-}$ sibling controls. The Rheb cKO female mice were fertile; their fecundity was indistinguishable from controls and they continued to have litters of normal size up to 18 weeks of age (Fig. $6 \mathrm{~A}$ and B). The male Rheb cKO mice, however, did not produce any offspring over a period of 20 weeks (Fig. 6C and D). This complete male infertility is expected given the oligoasthenoteratozoospermia observed above.

\section{Histomorphometric analyses of gametogenesis expose a defect in meiotic progression}

As an initial characterization of the disrupted seminiferous tubules, and to identify potential developmental blocks in spermatogenesis, cell counts were carried out to quantify the numbers of spermatogonia, spermatocytes, round spermatids, and elongated spermatids (Fig. 7A). Foxo1 expression was used as a convenient marker of undifferentiated spermatogonia (Goertz et al. 2011, Tarnawa et al. 2013). Immunostaining and counts revealed normal numbers of undifferentiated spermatogonia in Vasa-Cre; Rheb ${ }^{f /-}$ testes at all time points (Fig. 7C). Spermatocytes were counted based on their
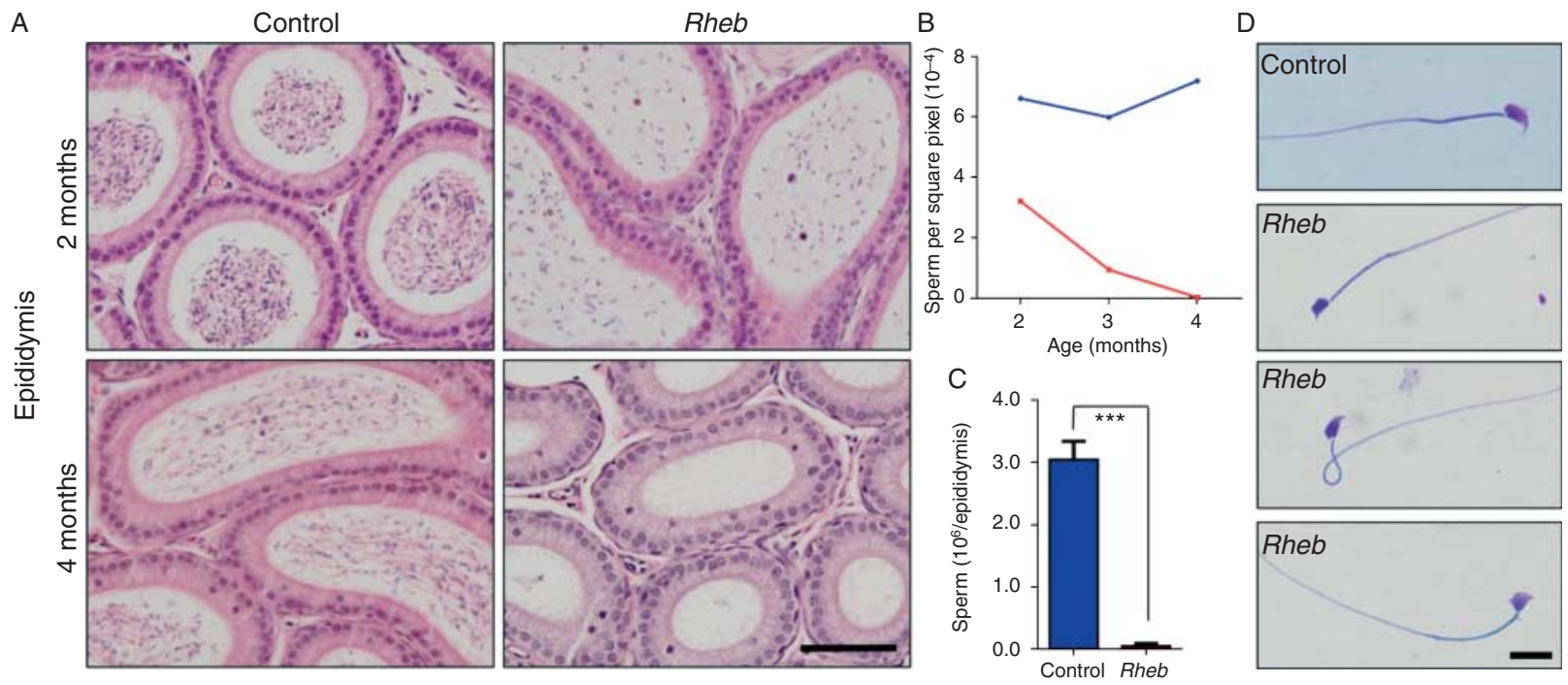

Figure 5 Total sperm production is drastically decreased and remaining sperm are malformed. (A) Histology of epididymides from control and experimental mice. Bar $=100 \mu \mathrm{m}$. (B) The decrease in epididymal sperm was quantified by sperm head density counts $(n=1$ for all time points and genotypes). (C) Total sperm counts were performed after releasing the sperm from the epididymis. ${ }^{* * *} P<0.0001$. Red, Vasa-Cre; Rheb ${ }^{f /}$, $n=4$ and blue, $R h e b^{f /-}, n=5$ sibling controls. (D) The few sperm obtained from the epididymis of Rheb cKO mice were all immotile and exhibited abnormal morphology. Bar $=10 \mu \mathrm{m}$; all panels at same magnification. 

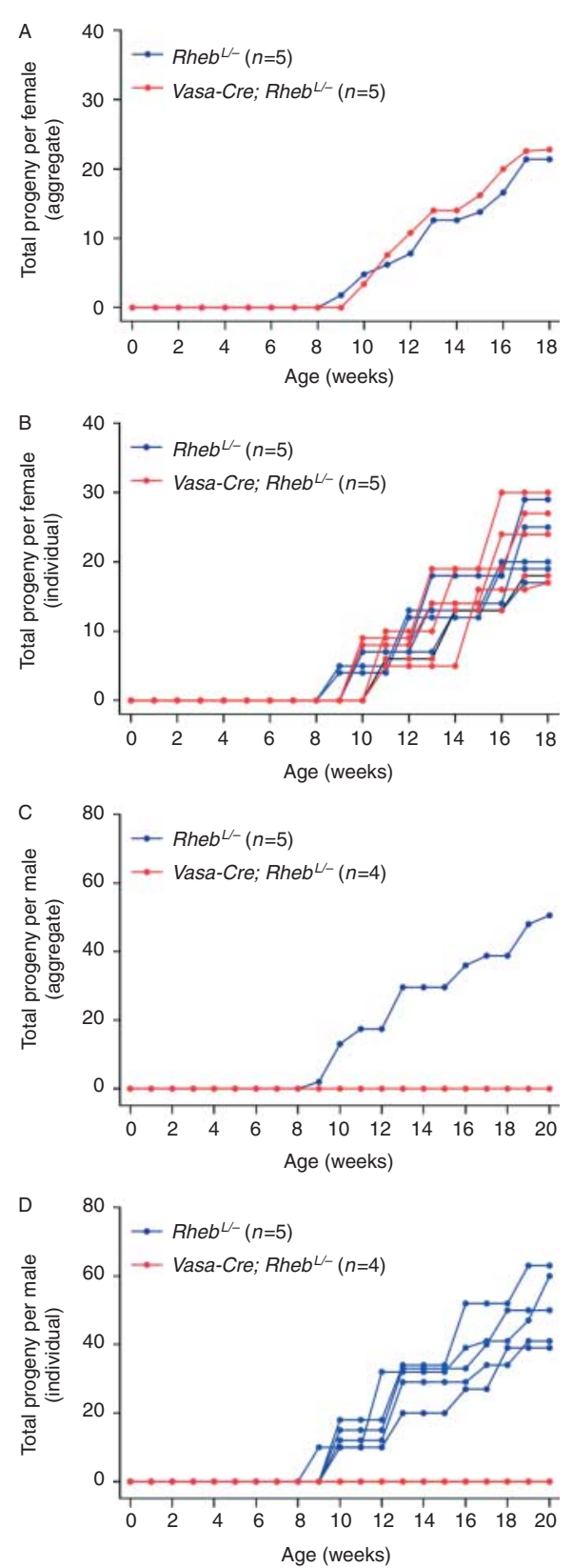

Figure 6 Serial breeding assays reveal that Rheb cKO males are sterile while Rheb cKO females are fertile with normal fecundity to 18 weeks of age. (A) Aggregate (i.e. all animals of the same genotype averaged) and (B) individual (i.e. every line represents a single animal) representations of total progeny per female. (C) Aggregate and (D) individual representations of total progeny per male.

large size and distinctive cellular morphology. A significant accumulation of spermatocytes was observed at 2 months but numbers reverted to normal at 3 and 4 month time points. By 5 months of age, a significant decrease in spermatocyte numbers was observed (Fig. 7D). Quantifications of round and elongated spermatids were performed through Crem1 immunostaining and cellular morphology respectively
(Delmas et al. 1993; Fig. 7B). Decreases in round and elongated spermatid counts were observed at early time points (Fig. 7C and D). Thus in summary, Rheb cKO males exhibited a striking age-dependent decrease in spermatid production and a more modest decrease in spermatocytes, while maintaining normal numbers of undifferentiated spermatogonia. These results point to abnormalities in meiotic progression.

\section{Spermatogonia continue to proliferate and initiate meiosis}

In light of the progressive decrease in spermatocytes in the Vasa-Cre; $R h e b^{f /-}$ mice, proliferation, and meiotic entry were further evaluated by immunostaining for Ki67 and phosphorylated $\mathrm{H} 2 \mathrm{AX}(\mathrm{pH} 2 \mathrm{AX})$ respectively. Ki67 is a marker of actively cycling cells, while $\mathrm{H} 2 \mathrm{AX}$ is phosphorylated in response to double-stranded DNA breaks (Hamer et al. 2002, Akbay et al. 2013). pH2AX nuclear foci serve as a marker of meiotic recombination in response to the formation of chiasma between sister chromatids in leptotene spermatocytes. However, detailed counts of cells positive for $\mathrm{Ki} 67$ and pH2AX foci revealed no significant differences between VasaCre; $R h e b^{f /-}$ and control males at all time points studied (Fig. 8). Therefore, spermatogonial proliferation and meiotic entry per se appears largely unaffected in Rheb cKO males, again consistent with a severe defect in meiotic progression.

\section{The activation status of $m T O R, S 6 K$, and $4 E B P$ appears to be unaffected in Vasa-Cre; Rheb ${ }^{\mathrm{f} /-}$ mice}

We then sought to determine the activation status of mTOR pathway components in Rheb cKO testes as well as ovaries. Immunohistochemistry was performed for phosphorylated (i.e. activated) mTOR pathway members p-mTOR, p-S6K, p-S6, and p-4EBP using well-validated phosphorylation site-specific antibodies for each of these factors. However, no significant differences were observed in the phosphorylation state of any of these mTOR pathway components in Rheb cKO vs control testes, as gauged by staining intensity, when specific spermatogenic stages were compared (Fig. 9).

\section{Discussion}

These studies document a male infertility phenotype associated with multiple defects in the meiotic and postmeiotic stages of spermatogenesis in mice with conditional genetic inactivation of Rheb in the germ line. Interestingly, given previous studies implicating mTORC1 in stem cell self-renewal, Rheb was dispensable for spermatogonial self-renewal and mitotic proliferation (Hobbs et al. 2010). However, Rheb was necessary for spermiogenesis, as Rheb males exhibited 
A

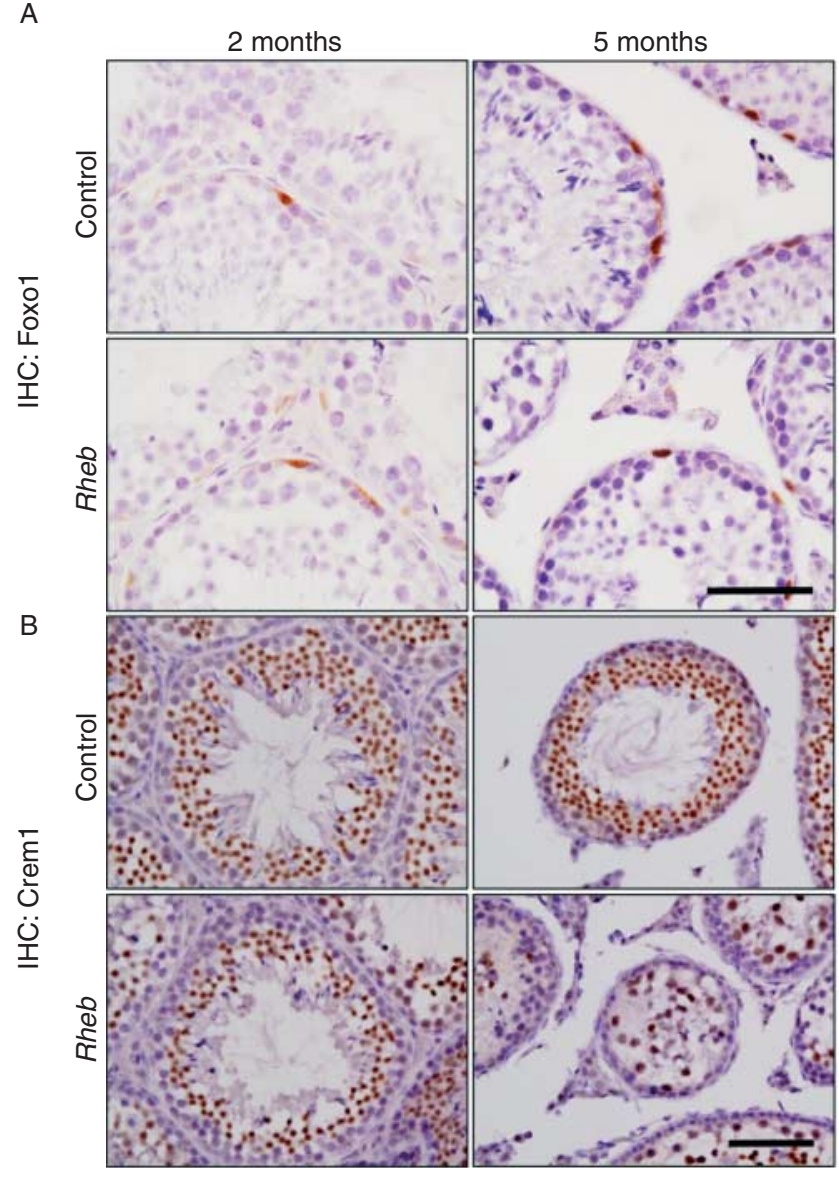

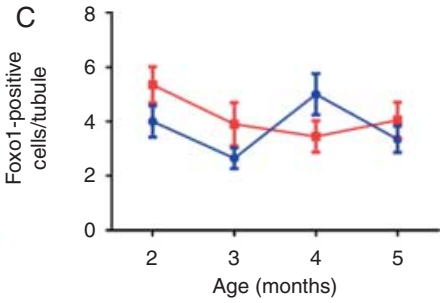
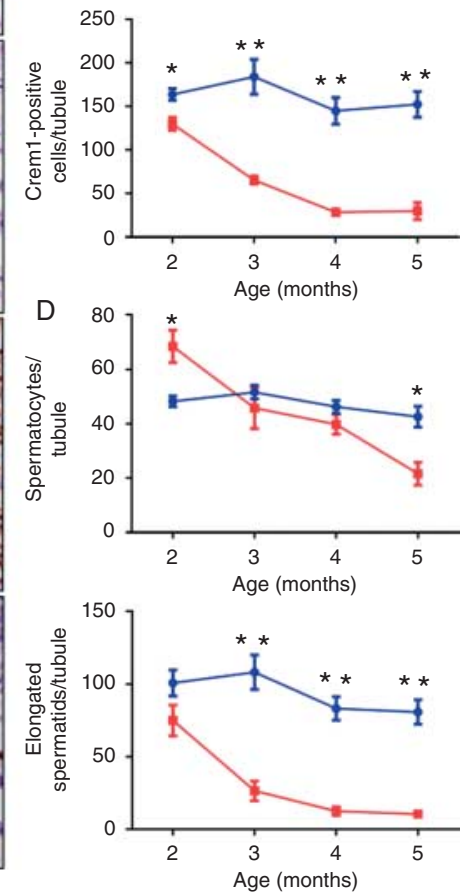

Figure 7 Cell counts reveal meiotic progression defect. (A) Foxo1 and (B) Crem1 immunostains of Rheb cKO (Vasa-Cre; Rheb ${ }^{f /-}$ ) and control testes at 2 and 5 months of age. Bar $=50$ and $100 \mu$ m respectively. (C) Quantification of Foxo1- and Crem1-positive cell counts in 20 tubules; $* P<0.05$ and ${ }^{* *} P<0.0005$ by unpaired $t$-test. (D) Counts of spermatocytes and spermatids in hematoxylin-stained tissue sections. The counts of elongated spermatids closely mirrored the observed decrease in Crem $1^{+}$round spermatids; ${ }^{*} P<0.05$ and ${ }^{* *} P<0.005$ by unpaired $t$-test.

greatly reduced epididymal sperm numbers, and the few sperm present were immotile and exhibited grossly abnormal head morphology. A delay in meiotic progression was also evident at 2 months of age as spermatocyte counts were significantly increased. Immunostains for meiotic initiation-associated doublestrand DNA breaks were relatively unaffected, further suggesting that these defects were due to delays or abnormalities in meiotic progression, and not meiotic initiation per se. Interestingly, these pleiotropic phenotypes were age-dependent, and in 5-month-old males, the meiotic defect was even more severe, with greatly decreased numbers of post-meiotic spermatids.

A large number of mouse mutants with combined defects in meiosis and spermiogenesis have been described. These mutations affect a wide range of processes, from acrosome and tail formation to misregulation of late spermiogenesis gene expression (Yan 2009). The multiple defects that we have described resemble in several respects the spermatogenesis phenotype previously described for mice with conditional germ line inactivation of Dicer, the miRNA-processing enzyme (Korhonen et al. 2011, Romero et al. 2011). The Dicer CKO is also infertile due to cumulative defects in meiosis and spermiogenesis that result in an oligoasthenoteratozoospermia phenotype. Delayed progression of meiosis was also observed, but the phenotype was manifested during the first wave of spermatogenesis and did not worsen with age (Romero et al. 2011). Both Rheb and Dicer control critical pathways regulating protein expression, albeit through two fundamentally different mechanisms. The biological basis of these Rheb and Dicer male infertility phenotypes probably resides in this precise regulation of gene expression and translation required to complete the complex transcription-independent processes of meiotic and post-meiotic sperm development. With hundreds of testis-specific genes expressed in spermatocytes and spermatids, the translation of these mRNAs must be perfectly orchestrated to coordinate acrosome biogenesis, tail formation, nuclear 


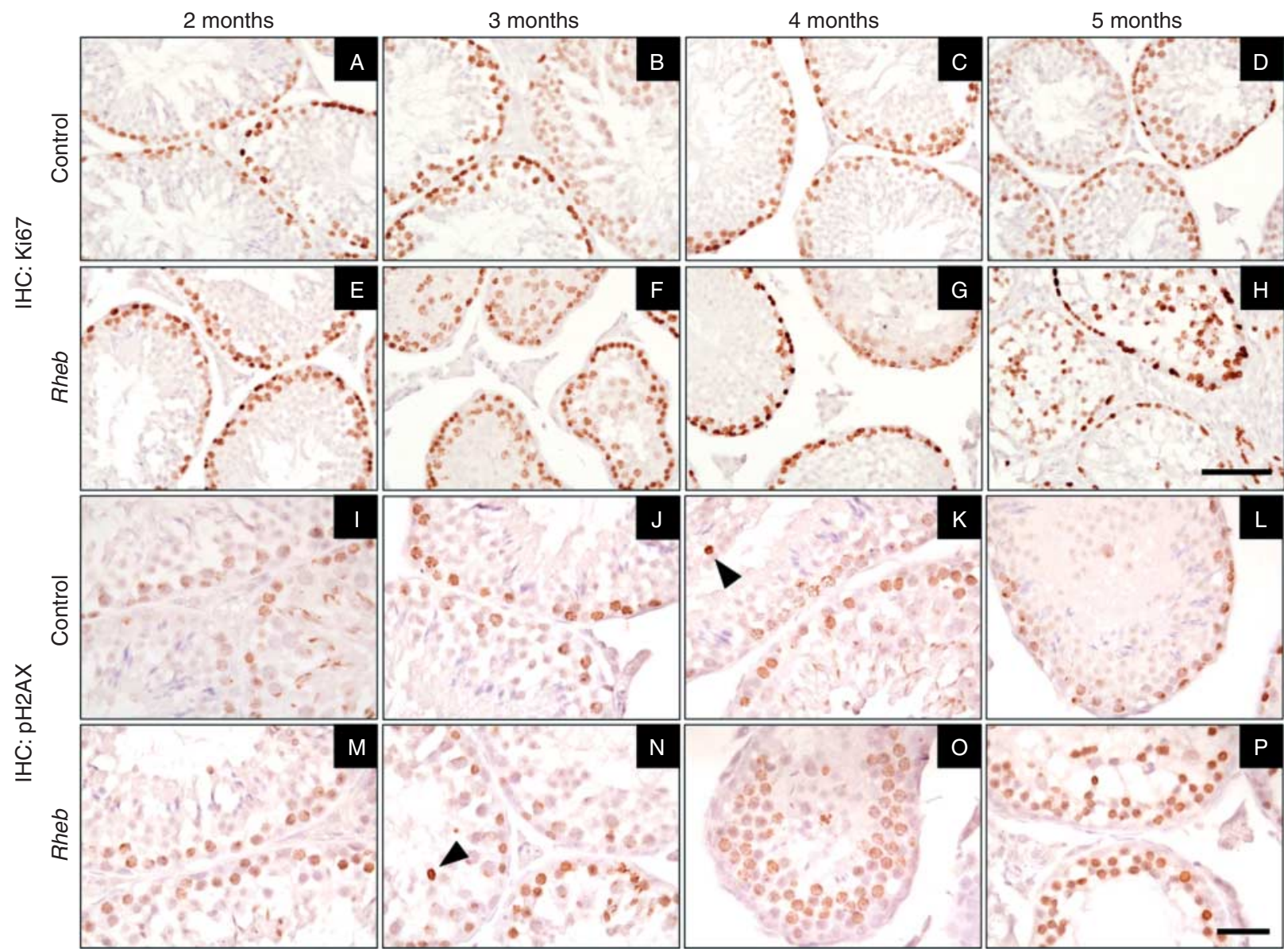

Figure 8 Analyses of cell proliferation and meiotic entry. (A, B, C, D, E, F, G and H) Immunostaining for Ki67 as a marker for cellular proliferation. Bar $=100 \mu \mathrm{m}$. (I, J, K, L, M, N, O and P) Immunostaining for $\mathrm{pH} 2 \mathrm{AX}$ as a marker of meiotic entry. Scattered apoptotic germ cells serve as an internal positive control (arrowheads) since apoptotic cells undergo DNA fragmentation and are strongly positive for $\mathrm{pH} 2 \mathrm{AX}$. $\mathrm{Bar}=25 \mu \mathrm{m}$.

condensation, and cytoplasm removal. With the loss-offunctional spermatozoa in Vasa-Cre; Rheb ${ }^{f /-}$ function in the testis, it is reasonable to hypothesize that regulation of protein translation by mTORC1 is required for spermiogenesis. Although we did not document an obvious defect in spermatogonial function, and the numbers of these cells were unaffected, it is possible that Rheb inactivation resulted in some unidentified abnormality (metabolic, transcriptional, etc.) in early stages of spermatogenesis that became manifest at later steps of spermatogenesis.

With regard to the control of protein translation, Rheb and mTORC1 act through canonical pathways including the phosphorylation of 4EBP and S6K. We were unable to document, by in situ methods or western blotting, significant alterations in the phosphorylation state of these key mTOR effectors. Several possibilities may be considered. First, by western blotting, the significant somatic cell contamination of whole testes lysates could make it difficult to detect differences in phosphorylation levels (we observed no differences through these methods - unpublished data). We also failed to detect differences in the phosphorylation levels in these factors (and hence significant alterations in mTORC1 signaling) by potentially more sensitive in situ methods, although immunohistochemistry may also be insensitive to relatively modest changes in target epitope levels. It is also possible that unknown feedback loops serve to preserve Tsc/mTOR signaling in the absence of Rheb. Although a precise molecular mechanism of action was not determined, the Rheb cKO is the first genetic in vivo study which implicates $R$ heb in the regulation of spermatogenesis.

Despite the complete sterility of Rheb cKO males, cKO females were fertile and exhibited normal fecundity. We observed no alterations in primordial follicle numbers, or follicle maturation, by careful histological analyses. This result is somewhat surprising in light of previous studies clearly demonstrating a central role of mTOR signaling in the regulation of primordial follicle activation. The global follicle activation phenotype previously documented in TSC1 or TsC2 CKO females was associated with dramatic upregulation of the mTOR signaling pathway (demonstrable with the same immunohistochemical assays performed in our study; 


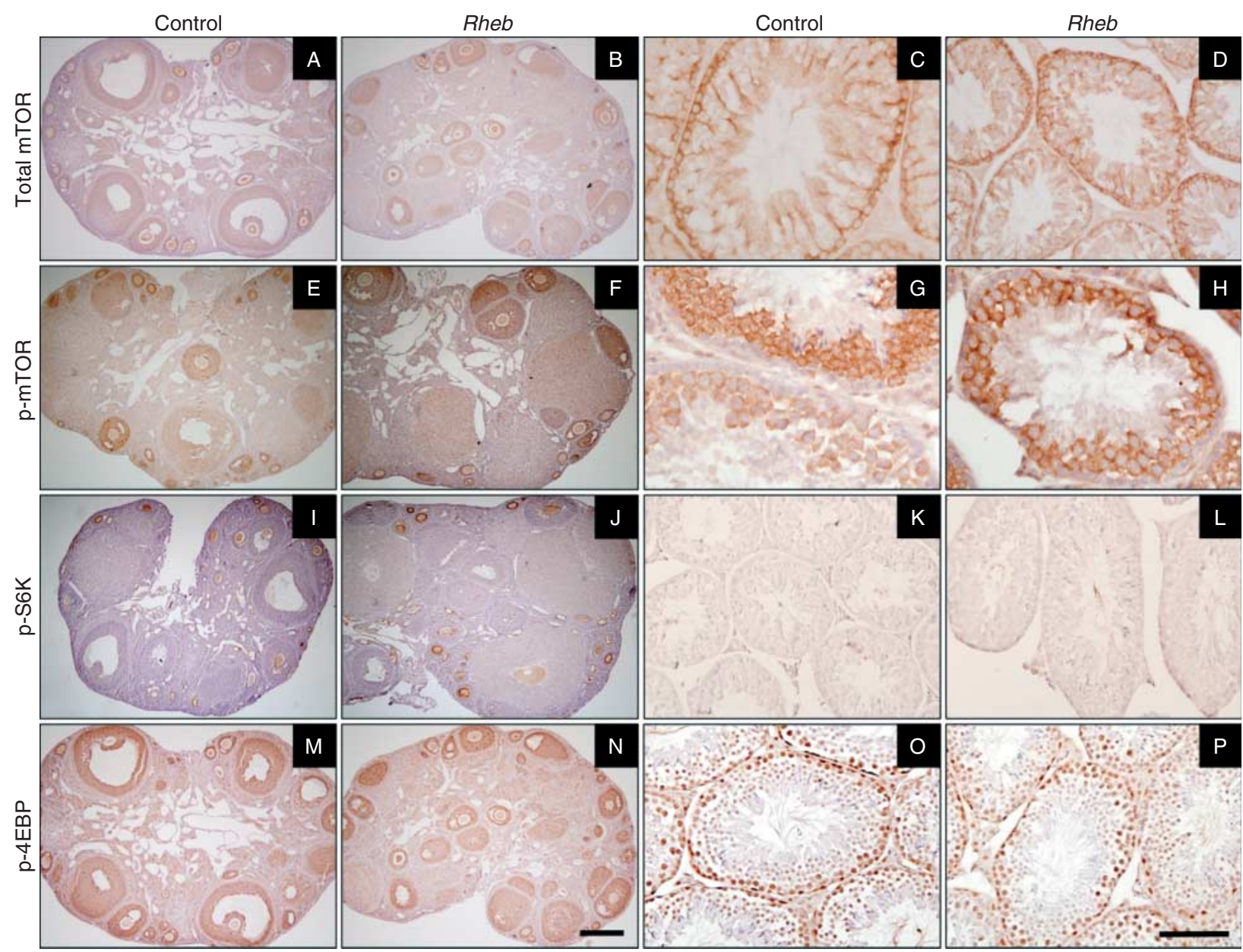

Figure 9 Analysis of mTOR and downstream effectors. Immunostains with phosphorylation site-specific antibodies against canonical mTOR pathway members in both ovaries (left) and testes (right). Bar $=250$ and $100 \mu \mathrm{m}$ respectively. (A, B, C and D) Total mTOR, (E, F, G and H) p-mTOR, (I, J, K and L) $\mathrm{p}-\mathrm{S} 6 \mathrm{~K}$, and (M, N, O and P) p-4EBP.

Adhikari et al. 2009, 2010), yet conditional inactivation of Rheb in oocytes did not have any observable effect on follicle maturation. Although different $\mathrm{Cre}$ deletor lines were used in these studies (Vasa-Cre and Gdf9-Cre), this does not appear to provide an adequate explanation, as both $\mathrm{Cre}$ deletor lines have been extensively utilized and well documented to lead to efficient Cre-mediated recombination in primordial oocytes (Castrillon et al. 2003, Lan et al. 2004, Gallardo et al. 2007a, Adhikari et al. 2009). If mTORC1 activation played an essential role in the recruitment of primordial follicles, Rheb inactivation would be predicted to result in a primordial follicle arrest phenotype in the absence of mTOR signaling secondary to Rheb inactivation. Explanations for the lack of phenotype may include the action, within the oocyte, of functionally redundant members of the GTPase superfamily, such as the Rheb homolog Rheb/1. Although global Rheb/1 KO mice were viable and fertile (Zou et al. 2011), we have documented Rheb/1 expression in the oocyte (Fig. 1C and D), which could serve to compensate for the failure of complete Rheb inactivation to affect any necessary thresholds of mTOR activation (Tee et al. 2005). A Rheb germ cell cKO in a Rheb/1-null background may provide insights into this hypothesis. Alternately, an unknown Rheb homolog, or perhaps a more distantly related Ras-like GTPase, may provide this function. Furthermore, cellular functions including the control of apoptosis, differentiation, and the actin cytoskeleton regulation have been proposed to be modulated by non-canonical (i.e. mTORC1-independent) functions of Tsc1/Tsc2 (Neuman \& Henske 2011) and it is possible that such mTOR-independent actions of the Tsc complex also contribute to the lack of a Rheb ovarian phenotype.

\section{Declaration of interest}

The authors declare that there is no conflict of interest that could be perceived as prejudicing the impartiality of the research reported. 


\section{Funding}

The project described was supported by Award Number R01HD048690 from the Eunice Kennedy Shriver National Institute of Child Health \& Human Development. The content is solely the responsibility of the authors and does not necessarily represent the official views of the Eunice Kennedy Shriver National Institute of Child Health \& Human Development or the National Institutes of Health.

\section{Acknowledgements}

The authors thank Paul F Worley (Johns Hopkins University School of Medicine) and Bo Xiao (Sichuan University) for the Rheb-floxed mice. They thank Delali Bassowou for technical assistance, as well as Kent Hamra and Rolf Brekken for helpful discussions.

\section{References}

Adhikari D, Flohr G, Gorre N, Shen Y, Yang H, Lundin E, Lan Z, Gambello MJ \& Liu K 2009 Disruption of Tsc2 in oocytes leads to overactivation of the entire pool of primordial follicles. Molecular Human Reproduction 15 765-770. (doi:10.1093/molehr/gap092)

Adhikari D, Zheng W, Shen Y, Gorre N, Hamalainen T, Cooney AJ, Huhtaniemi I, Lan ZJ \& Liu K 2010 Tsc/mTORC1 signaling in oocytes governs the quiescence and activation of primordial follicles. Human Molecular Genetics 19 397-410. (doi:10.1093/hmg/ddp483)

Akbay EA, Pena CG, Ruder D, Michel JA, Nakada Y, Pathak S, Multani AS, Chang S \& Castrillon DH 2013 Cooperation between p53 and the telomere-protecting shelterin component Pot1a in endometrial carcinogenesis. Oncogene 32 2211-2219. (doi:10.1038/onc.2012.232)

Castrillon DH, Miao L, Kollipara R, Horner JW \& DePinho RA 2003 Suppression of ovarian follicle activation in mice by the transcription factor Foxo3a. Science 301 215-218. (doi:10.1126/science. 1086336)

Contreras CM, Akbay EA, Gallardo TD, Haynie JM, Sharma S, Tagao O, Bardeesy N, Takahashi M, Settleman J, Wong KK et al. 2010 Lkb1 inactivation is sufficient to drive endometrial cancers that are aggressive yet highly responsive to mTOR inhibitor monotherapy. Disease Models \& Mechanisms 3 181-193. (doi:10.1242/dmm. 004440)

Delmas V, van der Hoorn F, Mellstrom B, Jegou B \& Sassone-Corsi P 1993 Induction of CREM activator proteins in spermatids: down-stream targets and implications for haploid germ cell differentiation. Molecular Endocrinology 7 1502-1514. (doi:10.1210/mend.7.11.8114765)

Fingar DC \& Blenis J 2004 Target of rapamycin (TOR): an integrator of nutrient and growth factor signals and coordinator of cell growth and cell cycle progression. Oncogene 23 3151-3171. (doi:10.1038/sj.onc. 1207542)

Gallardo T, Shirley L, John GB \& Castrillon DH 2007 a Generation of a germ cell-specific mouse transgenic Cre line, Vasa-Cre. Genesis 45 413-417. (doi:10.1002/dvg.20310)

Gallardo TD, John GB, Shirley L, Contreras CM, Akbay EA, Haynie JM, Ward SE, Shidler MJ \& Castrillon DH 2007 b Genomewide discovery and classification of candidate ovarian fertility genes in the mouse. Genetics 177 179-194. (doi:10.1534/genetics.107.074823)

Gallardo TD, John GB, Bradshaw K, Welt C, Reijo-Pera R, Vogt PH, Touraine P, Bione S, Toniolo D, Nelson LM et al. 2008 Sequence variation at the human FOXO3 locus: a study of premature ovarian failure and primary amenorrhea. Human Reproduction 23 216-221. (doi:10.1093/humrep/dem255)

Goertz MJ, Wu Z, Gallardo TD, Hamra FK \& Castrillon DH 2011 Foxo1 is required in mouse spermatogonial stem cells for their maintenance and the initiation of spermatogenesis. Journal of Clinical Investigation $\mathbf{1 2 1}$ 3456-3466. (doi:10.1172/JCI57984)
Hamer G, Roepers-Gajadien H, Duyn-Goedhart A, Gademan I, Kal H, Buul P \& Rooij D 2002 DNA double-strand breaks and $\gamma-\mathrm{H} 2 \mathrm{AX}$ signaling in the testis. Biology of Reproduction 68 628-634. (doi:10.1095/ biolreprod.102.008672)

Hobbs RM, Seandel M, Falciatori I, Rafii S \& Pandolfi PP 2010 Plzf regulates germline progenitor self-renewal by opposing mTORC1. Cell 142 468-479. (doi:10.1016/j.cell.2010.06.041)

Hosaka T, Biggs WH III, Tieu D, Boyer AD, Varki NM, Cavenee WK \& Arden KC 2004 Disruption of forkhead transcription factor (FOXO) family members in mice reveals their functional diversification. PNAS 101 2975-2980. (doi:10.1073/pnas.0400093101)

John GB, Shirley LJ, Gallardo TD \& Castrillon DH 2007 Specificity of the requirement for Foxo3 in primordial follicle activation. Reproduction 133 855-863. (doi:10.1530/REP-06-0051)

John GB, Gallardo TD, Shirley LJ \& Castrillon DH 2008 Foxo3 is a PI3K-dependent molecular switch controlling the initiation of oocyte growth. Developmental Biology 321 197-204. (doi:10.1016/j.ydbio. 2008.06.017)

Korhonen HM, Meikar O, Yadav RP, Papaioannou MD, Romero Y, Da Ros M, Herrera PL, Toppari J, Nef S \& Kotaja N 2011 Dicer is required for haploid male germ cell differentiation in mice. PLOS ONE 6 e24821. (doi:10.1371/journal.pone.0024821)

Lan ZJ, Xu X \& Cooney AJ 2004 Differential oocyte-specific expression of Cre recombinase activity in GDF-9-iCre, Zp3cre, and Msx2Cre transgenic mice. Biology of Reproduction 71 1469-1474. (doi:10.1095/ biolreprod.104.031757)

Laplante M \& Sabatini DM 2009 mTOR signaling at a glance. Journal of Cell Science 122 3589-3594. (doi:10.1242/jcs.051011)

Neuman NA \& Henske EP 2011 Non-canonical functions of the tuberous sclerosis complex-Rheb signalling axis. EMBO Molecular Medicine 3 189-200. (doi:10.1002/emmm.201100131)

Reddy P, Liu L, Adhikari D, Jagarlamudi K, Rajareddy S, Shen Y, Du C, Tang W, Hamalainen T, Peng SL et al. 2008 Oocyte-specific deletion of Pten causes premature activation of the primordial follicle pool. Science 319 611-613. (doi:10.1126/science.1152257)

Romero Y, Meikar O, Papaioannou MD, Conne B, Grey C, Weier M, Pralong F, De Massy B, Kaessmann H, Vassalli JD et al. 2011 Dicer1 depletion in male germ cells leads to infertility due to cumulative meiotic and spermiogenic defects. PLOS ONE 6 e25241. (doi:10.1371/journal. pone.0025241)

Schneider CA, Rasband WS \& Eliceiri KW 2012 NIH Image to ImageJ: 25 years of image analysis. Nature Methods 9 671-675. (doi:10.1038/ nmeth.2089)

Sullivan SD \& Castrillon DH 2011 Insights into primary ovarian insufficiency through genetically engineered mouse models. Seminars in Reproductive Medicine 29 283-298. (doi:10.1055/s-0031-1280914)

Tarnawa ED, Baker MD, Aloisio GM, Carr BR \& Castrillon DH 2013 Gonadal expression of foxo1, but not foxo3, is conserved in diverse mammalian species. Biology of Reproduction 88 103. (doi:10.1095/ biolreprod.112.105791)

Tee AR, Blenis J \& Proud CG 2005 Analysis of mTOR signaling by the small G-proteins, Rheb and RhebL1. FEBS Letters 579 4763-4768. (doi:10.1016/j.febslet.2005.07.054)

Tzivion G, Dobson M \& Ramakrishnan G 2011 FoxO transcription factors; Regulation by AKT and 14-3-3 proteins. Biochimica et Biophysica Acta 1813 1938-1945. (doi:10.1016/j.bbamcr.2011.06.002)

Wyrobek AJ \& Bruce WR 1975 Chemical induction of sperm abnormalities in mice. PNAS 72 4425-4429. (doi:10.1073/pnas.72.11.4425)

Yan W 2009 Male infertility caused by spermiogenic defects: lessons from gene knockouts. Molecular and Cellular Endocrinology 306 24-32. (doi:10.1016/j.mce.2009.03.003)

Zou J, Zhou L, Du XX, Ji Y, Xu J, Tian J, Jiang W, Zou Y, Yu S, Gan L et al. 2011 Rheb1 is required for mTORC1 and myelination in postnatal brain development. Developmental Cell 20 97-108. (doi:10.1016/j.devcel. 2010.11.020)

Received 11 July 2013

First decision 20 August 2013

Revised manuscript received 19 December 2013

Accepted 21 January 2014 